Accepted after revision: February 16, 2011

\title{
Morphometric Differences between the Medial and Lateral Meniscus in Healthy Men - A Three-Dimensional Analysis Using Magnetic Resonance Imaging
}

\author{
K. Bloecker ${ }^{a}$ W. Wirth ${ }^{a, b} \quad$ M. Hudelmaier ${ }^{a, b} \quad$ R. Burgkart ${ }^{c} \quad$ R. Frobell ${ }^{a, d}$ \\ F. Eckstein ${ }^{a, b}$ \\ anstitute of Anatomy and Musculoskeletal Research, Paracelsus Medical University, Salzburg, Austria; \\ ${ }^{b}$ Chondrometrics $\mathrm{GmbH}$, Ainring, and ${ }^{\mathrm{C} C l i n i c}$ for Orthopedics and Traumatology, Technical University of Munich, \\ Munich, Germany; ${ }^{\text {d}}$ Department of Orthopedics, Clinical Sciences, Lund University, Lund, Sweden
}

\section{Key Words}

Knee joint $\cdot$ Magnetic resonance imaging $\cdot$ Meniscus •

Morphometry

\begin{abstract}
The objective of this work was to characterize tibial plateau coverage and morphometric differences of the medial (MM) and lateral meniscus (LM) in a male reference cohort using three-dimensional imaging. Coronal multiplanar reconstructions of a sagittal double-echo steady state with water excitation magnetic resonance sequence (slice thickness: 1.5 $\mathrm{mm}$, and in-plane resolution: $0.37 \times 0.70 \mathrm{~mm}$ ) were analyzed in 47 male participants without symptoms, signs or risk factors of knee osteoarthritis of the reference cohort of the Osteoarthritis Initiative. The medial and lateral tibial (LT) plateau cartilage area and the tibial, femoral and external surfaces of the MM and LM were manually segmented throughout the entire knee. This process was assisted by parallel inspection of a coronal intermediately weighted turbo spin echo sequence. Measures of tibial coverage, meniscus size, and meniscus position were computed three-dimensionally for the total menisci, the body, and the anterior and the posterior horn. The LM was found to cover a significantly greater $(p<0.001)$ proportion of the LT plateau $(59 \pm 6.8 \%)$ than the MM of the medial plateau (50 $\pm 5.5 \%)$. Whereas the vol-
\end{abstract}

ume of both menisci was similar (2.444 vs. $2.438 \mathrm{ml} ; \mathrm{p}=0.92)$, the LM displayed larger tibial and femoral surface areas $(p<$ $0.05)$ and a smaller maximal (7.2 \pm 1.0 vs. $7.7 \pm 1.1 \mathrm{~mm} ; \mathrm{p}<$ $0.01)$ and mean thickness ( $2.7 \pm 0.3$ vs. $2.8 \pm 0.3 \mathrm{~mm} ; \mathrm{p}<$ 0.001 ) than the medial one. Also, the LM displayed less (phys-

\begin{tabular}{ll}
\hline Abbreviations used in this paper \\
\hline 3D & $\begin{array}{l}\text { three-dimensional } \\
\text { area of cartilage } \\
\text { AC }\end{array}$ \\
ACdAB & $\begin{array}{l}\text { subchondral bone } \\
\text { denuded areas of subchondral bone }\end{array}$ \\
dAB & double-echo steady state \\
DESS & double-echo steady-state sequence with water excitation \\
DESSwe & external area of the meniscus \\
EA & femoral area of the meniscus \\
FA & intermediately weighted \\
IW & lateral meniscus \\
LM & lateral tibia \\
LT & medial meniscus \\
MM & magnetic resonance \\
MR & magnetic resonance image \\
MRI & medial tibia \\
MT & osteoarthritis \\
OA & Osteoarthritis Initiative \\
OAI & standard deviation \\
SD & tibial area of the meniscus \\
TA & turbo spin echo
\end{tabular}

\section{KARGER}

Fax +41613061234 E-Mail karger@karger.ch www.karger.com
Dr. Katja Bloecker

Institute of Anatomy and Musculoskeletal Research

Paracelsus Medical University

Strubergasse 21, AT-5020 Salzburg (Austria)

Tel. +43 662442002 1245, E-Mail katja.bloecker@pmu.ac.at 
iological) extrusion than the medial one. These data may guide strategies for meniscal tissue engineering and transplantation aiming to restore normal joint conditions.

Copyright $\odot 2011$ S. Karger AG, Basel

\section{Introduction}

The medial (MM) and lateral meniscus (LM) develop as fibrocartilaginous structures between the tibial and femoral articular surfaces of the knee joint [Doskocil, 1984; Fukazawa et al., 2009] and display only small postnatal histological changes [Meller et al., 2009]. It is commonly assumed that the menisci develop as discoid structures, but a recent study in 106 fetal human knee joints found that the medial tibial (MT) plateau was incompletely covered by the MM during all gestational stages from week 14 to 34 [Murlimanju et al., 2010]. Whereas a discoid LM is a relatively frequent (but often asymptomatic) finding, particularly in the Japanese population [Kramer and Micheli, 2009], a discoid MM is an extremely rare observation [Tachibana et al., 2003; Vidyadhara et al., 2006; Lee et al., 2007].

At week 14, the size of the fetal meniscus and tibial plateau areas were reported to be similar in the medial and lateral femorotibial compartment [Fukazawa et al., 2009]. Whereas the increase in size of the meniscus during growth was similar medially and laterally, the tibial plateau area increased more rapidly in the medial than in the lateral compartment [Fukazawa et al., 2009]; for this reason, the MM was found to cover a relatively smaller proportion of the MT area than the lateral one [of the lateral tibial (LT) area] at later gestational stages and in adults [Fukazawa et al., 2009].

Functionally, the menisci distribute loads, guide movement, reduce contact stress, and protect the articular cartilage during dynamic and static joint loading [Walker and Erkman, 1975; Kurosawa et al., 1980; Messner and Gao, 1998; Eisenhart-Rothe et al., 2004; Chivers and Howitt, 2009]. The menisci were also reported to have proprioceptive and lubricating functions [Chivers and Howitt, 2009].

Macroscopically, the MM has been described as being crescent, sickle, C, sided U and sided V shaped, incomplete discoid or (almost) completely discoid shaped prenatally [Murlimanju et al., 2010]. In the adult, the MM is generally addressed as being $\mathrm{C}$ shaped and the $\mathrm{LM}$ as $\mathrm{O}$ shaped, with the MM being thinner and larger, and the lateral one smaller and thicker [Chivers and Howitt, 2009]. The anterior horns of both menisci were found to be narrower than the posterior horns [Messner and Gao, 1998].

Microscopically, three distinct layers were described in the meniscus cross section [Petersen and Tillmann, 1998]: (1) a meshwork of thin fibrils with a diameter of approximately $30 \mathrm{~nm}$ covering the tibial and femoral sides of the meniscus surfaces; (2) a layer of lamella-like collagen fibril bundles on the tibial and femoral surface beneath this superficial network; (3) the main portion of the meniscus collagen fibrils located in the central region and orientated in a circular manner, providing a functional explanation for the longitudinal orientation of the majority of tears in the meniscus tissue [Petersen and Tillmann, 1998].

Meniscus extrusion (outward displacement in radial direction towards the periphery) has been suggested to be considered a pathologic condition, when the external margin of the meniscus exceeds the margin of the tibial plateau by $>3 \mathrm{~mm}$ [Costa et al., 2004]. This condition has been shown to frequently co-occur with meniscal (specifically root) tears [Costa et al., 2004; Lerer et al., 2004; Rennie and Finlay, 2006; Magee, 2008]. In epidemiological studies, meniscus extrusion (usually graded visually) and tears were found to be significantly associated with the incidence [Englund et al., 2009] and progression [Hunter et al., 2006] of knee osteoarthritis (OA; i.e. cartilage loss), but no consistent association was found between meniscal extrusion and symptoms [Conaghan and Felson, 2004; Kornaat et al., 2006; Torres et al., 2006].

To date there exist little to no quantitative data on the amount of coverage of the MT and LT plateau (by the meniscus), meniscus size (thickness, areas and width) and physiological amounts of meniscal extrusion. However, these data could be important in the context of meniscal tissue engineering and transplantation [Stone et al., 1994; Pollard et al., 1995; Haut et al., 1998; Glowacki 2001; McDermott et al., 2004; Stone et al., 2007; Baker et al., 2009; Van Thiel et al., 2009; Yang and Temenoff, 2009; Elsner et al., 2010], which aim to restore normal (physiological) joint conditions.

Recently, we [Wirth et al., 2010a] and others [Elsner et al., 2010; Swanson et al., 2010] developed techniques for quantitative measurement of meniscus morphology from magnetic resonance (MR) images (MRI), specifically, software for determining three-dimensional (3D) measures of tibial plateau coverage, meniscus size, and relative meniscus position (extrusion) for the total meniscus as well as its subregions (i.e. the body, the anterior and the posterior horns) [Wirth et al., 2010a]. In the current study, we applied this technology to men from the Osteoar- 
thritis Initiative (OAI) (http://www.oai.ucsf.edu/datarelease/) who were without symptoms, signs or risk factors of knee OA.

Specifically, we addressed the following questions:

(1) How much of the tibial plateau is covered by the MM and LM, respectively?

(2) What is the size (volume, surface area, thickness and width) of the menisci (and their subregions), and do these measures differ between the medial and lateral side?

(3) What is the physiological (normal) magnitude of meniscus extrusion in the medial and lateral compartment, respectively?

\section{Subjects and Methods}

\section{Study Participants}

The image data from the male reference cohort of the OAI were used for this study. The OAI is a large ongoing cohort study targeted at characterizing risk factors associated with the onset and progression of symptomatic knee OA and at identifying (imaging) biomarkers of the disease. Of the 4,796 OAI participants, 122 were recruited as a reference subcohort (public-use data set $0 . F .1$ ), of which 47 were men and 75 women. Generally, participants were recruited at the University of Maryland School of Medicine (Baltimore), the Ohio State University (Columbus), the University of Pittsburgh and the Memorial Hospital of Rhode Island (Pawtucket). Informed consent was obtained from all participants and the study was approved by the local ethics committees.

Participants of the OAI reference cohort were considered 'healthy' in the sense that they had no symptoms or signs of knee $\mathrm{OA}$ and that they were not exposed to common risk factors of knee OA. Specifically they showed:

- no pain, aching or stiffness in either knee in the past year;

- no radiographic findings of femorotibial OA (osteophyte grade 0 and joint space narrowing grade 0 according to the Osteoarthritis Research Society International) of either knee using the clinic reading of the baseline bilateral fixed flexion radiographs [Peterfy et al., 2003], and

- no risk factors for the onset of knee OA, such as

- obesity defined as a body weight of $>170 \mathrm{lbs}(77.1 \mathrm{~kg})$ in women aged $45-69,>180 \mathrm{lbs}(81.7 \mathrm{~kg})$ in women aged 70 $79,>205 \mathrm{lbs}(93 \mathrm{~kg})$ in men aged 45-69, and >215 lbs $(97.5$ $\mathrm{kg}$ ) in men aged 70-79;

- a history of knee injury, defined as having caused difficulty in walking for at least 1 week;

- knee surgery;

- a family history of total knee replacement therapy in a biological parent or sibling;

- Heberden's nodes, defined as self-reported bony enlargements of one or more distal interphalangeal joints in both hands, and

- repetitive knee bending, defined as current daily activity at work or outside work, requiring frequent climbing, stooping, bending, lifting, squatting or kneeling.
The 47 men studied here were $57 \pm 9$ years (range $45-79$ years) old, had a body height of $1.74 \pm 0.07 \mathrm{~m}$, a weight of $79.2 \pm 8.2 \mathrm{~kg}$, and a body mass index of $26.1 \pm 2.9$.

\section{MR Imaging and Segmentation}

For the current study, publicly available MRI were used that had been acquired with a 3-tesla MAGNETOM Trio (Siemens, Erlangen, Germany) and quadrature transmit-receive knee coils (USA Instruments, Aurora, Ohio, USA), following the standardized OAI protocol [Peterfy et al., 2008; Schneider et al., 2008]. Specifically, the coronal multiplanar reconstructions of the sagittal double-echo steady-state (DESS) sequence with water excitation (DESSwe: repetition time $=16.3 \mathrm{~ms}$, echo time $=4.7 \mathrm{~ms}$, flip angle $=25^{\circ}$, reconstructed slice thickness $=1.5 \mathrm{~mm}$ and in-plane resolution $0.37 \times 0.7 \mathrm{~mm}$, interpolated to $0.37 \times 0.37 \mathrm{~mm}$ ) [Hardy et al., 1996; Eckstein et al., 2006b; Eckstein et al., 2007; Wirth et al., 2010b] of the right knees were used (fig. 1, 2). In addition, visual information was used from a 2-dimensional coronal intermediately weighted (IW) turbo spin echo (TSE) sequence (repetition time $=\mathrm{TR}=3,700 \mathrm{~ms}$; echo time $=\mathrm{TE}=29 \mathrm{~ms}$; slice thickness $=3 \mathrm{~mm}$ and in-plane resolution $0.37 \times 0.46 \mathrm{~mm}$, which was interpolated to match the slice thickness of the multiplanar reconstructed DESSwe. Further details on the MR imaging protocol have been given by Peterfy et al. [2008].

The MRI initially underwent quality control by an expert in quality control and in the use of the specific imaging software (R.F.). Manual segmentation was then performed by a single operator (K.B.) using specialized image analysis software (Chondrometrics $\mathrm{GmbH}$, Ainring, Germany) [Wirth et al., 2010a]. In both the medial and lateral femorotibial compartment, 4 tissue boundaries were segmented manually using the DESSwe (fig. 1, 2):

(1) the bearing surface of the tibial condyles (i.e. the tibial plateau area), specifically the area of the cartilage surface (AC, according to an accepted cartilage morphometry nomenclature [Eckstein et al., 2006a] of the medial and lateral condyle (MT and LT); please note that this interface is termed ACdAB, as it may also include denuded areas of subchondral bone (dAB), but not osteophytes, in pathological cases [Wirth et al., 2010a] (fig. 1-4);

(2) the tibial area of the MM and LM = TA = the area of the meniscus facing the tibial cartilage surface (fig. 1, 4);

(3) the femoral area of $\mathrm{MM}$ and $\mathrm{LM}=\mathrm{FA}=$ the area of the meniscus facing the femoral cartilage surface (fig. 1, 4), and

(4) the external area of MM and $\mathrm{LM}=\mathrm{EA}=$ the area of the meniscus, facing the joint capsule (fig. 1, 4).

In order to improve segmentation, sample series of coronal anatomical knee joint sections that were integrated with the software were used as a reference, as well as figures published in a series of articles on sectional anatomy of the knee joint [Burgkart et al., 1995]. In addition, in the first half of the cohort, the coronal IW TSE images were used in the meniscal segmentation, since these images are commonly used to evaluate meniscus pathology [Roemer et al., 2005; Guermazi et al., 2008; Roemer et al., 2009]. The time required for segmenting the MM and LM varied between 45 and $90 \mathrm{~min}$.

The nomenclature for meniscal measures used here was adopted from a previous paper [Wirth et al., 2010a], which itself was based on a proposal on cartilage morphometry measures [Eckstein et al., 2006a]. Anteriorly and posteriorly, segmentation started (or ended) where both the tibial cartilage and the menis- 

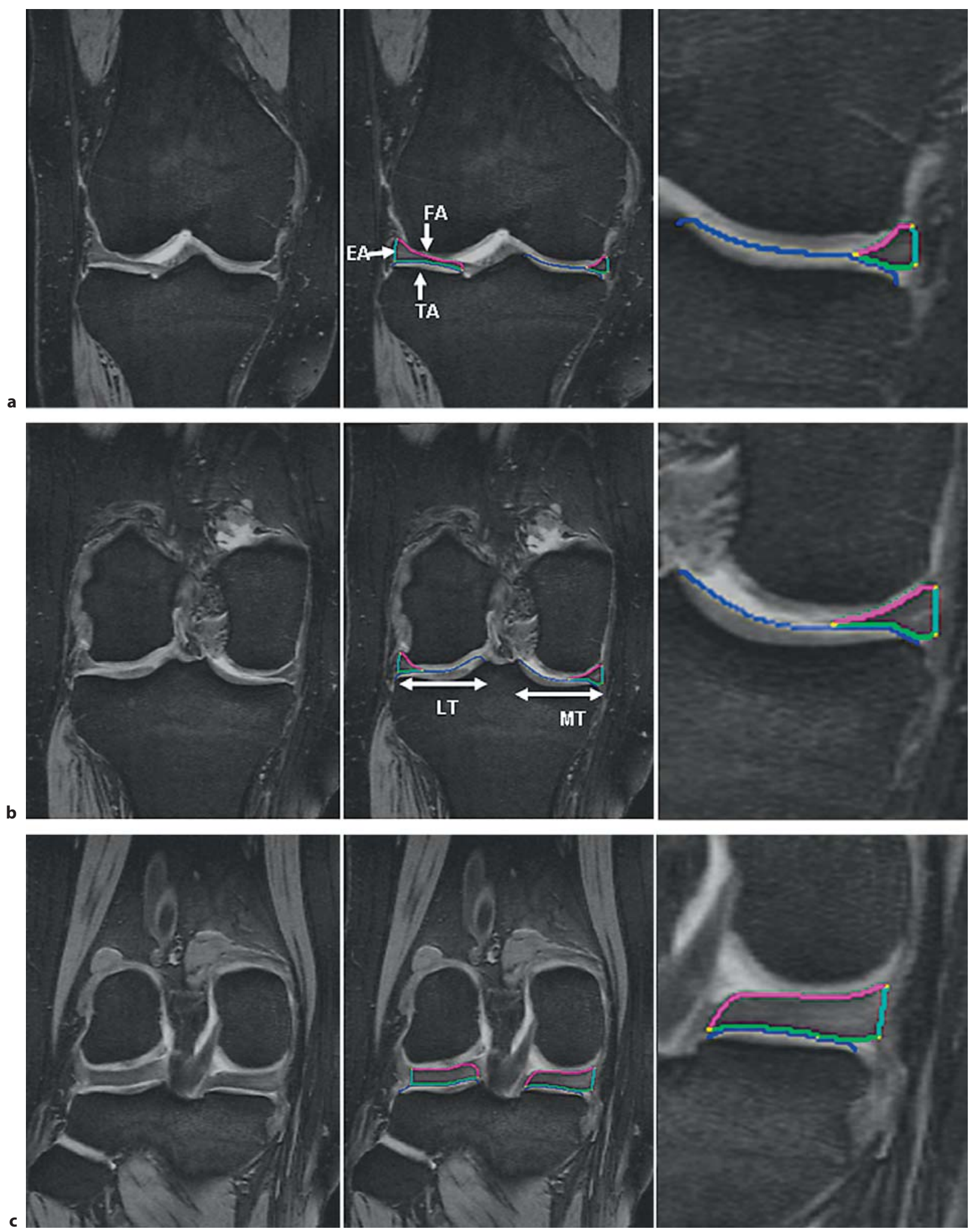

Fig. 1. Coronal MRI of the right knee without segmentation (left), with segmentation of the MM and LM (middle), and with the segmentation of the MM zoomed (right). The tibial plateau area (ACdAB) is shown in blue, the TA in green, the FA in magenta, and the EA in turquoise. a MRI at the level of the anterior horns. $\mathbf{b}$ MRI showing the meniscal body. c MRI at the level of the posterior horns. 

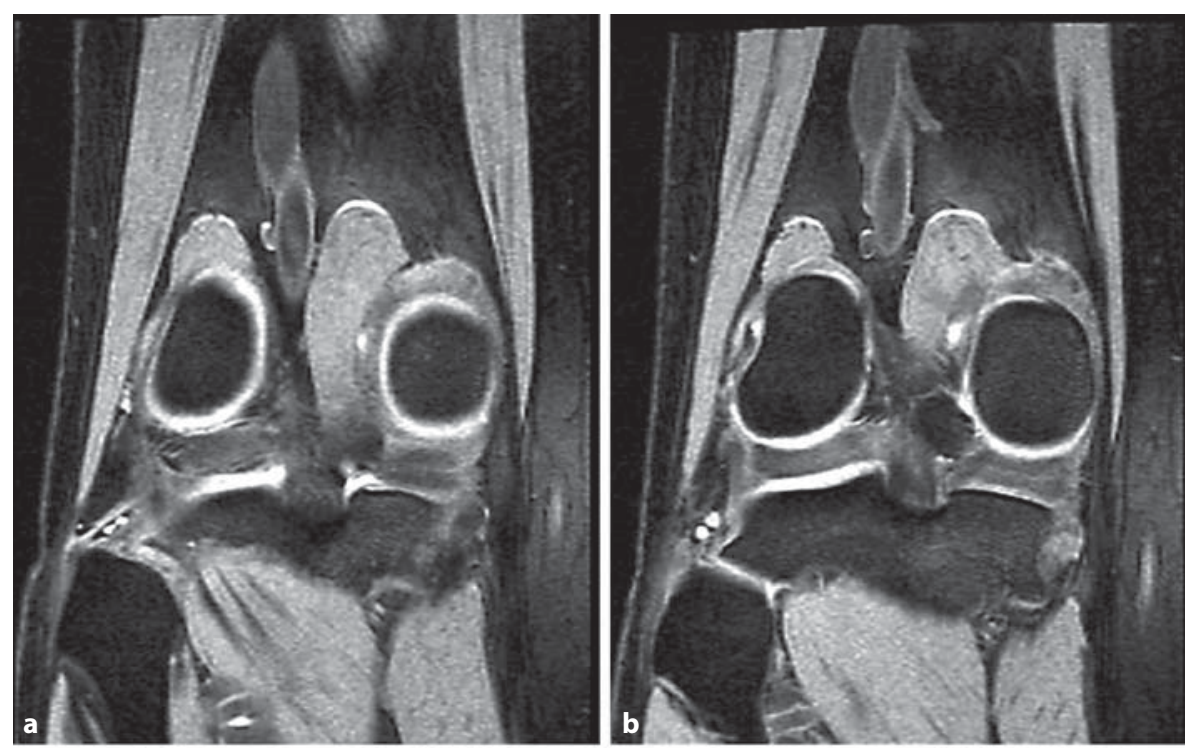

Fig. 2. Coronal MRI showing the anterior and posterior limits of meniscus segmentation. a MRI slice posterior to the MM, which was not segmented, because the MT plateau or the MM could not be reliably delineated. b First MRI slice in which the MM and MT plateau was segmented. Segmentation is not shown for better clarity. c Last MRI slice in which the MM and MT plateau was segmented. Segmentation is not shown for better clarity. d MRI slice anterior to the MM, which was not segmented, because the MT plateau or the MM could not be reliably delineated.
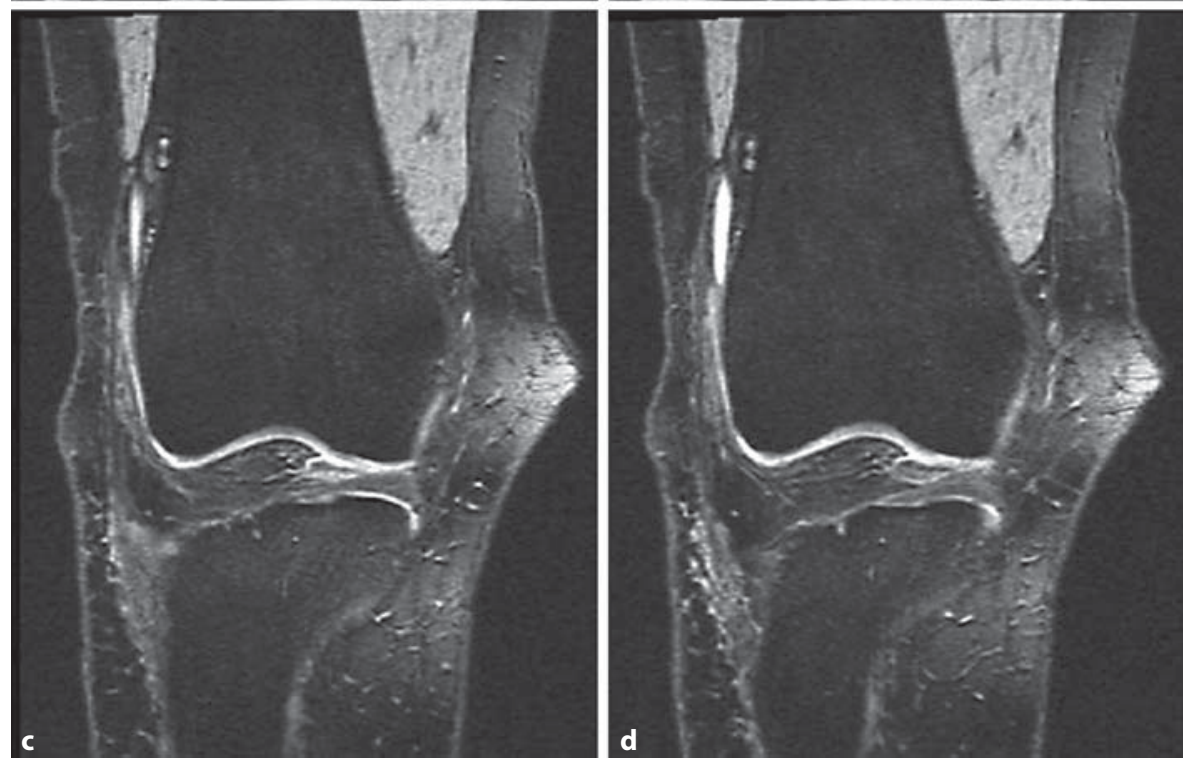

cus could be reliably identified (fig. 2); hence, anterior or posterior meniscus extrusion could not be detected from the coronal imaging protocol [Hunter et al., 2006] due to partial volume effect of the coronal images. Internally, the MM and LM borders were defined by the internal margin of the cartilage surface of MT and LT as anatomical landmarks, because the anterior and posterior horns are continuous with the transverse and meniscofemoral ligament (and with the common bony insertions of the cruciate ligament), and because no intrinsic anatomical demarcation can be used to separate these structures. For this reason, the TA that is not covered by ACdAB can be used as a measure of meniscal extrusion (see below). The segmentations were reviewed by an expert in the quality control of cartilage and meniscus segmentations (F.E.), and adjustments were made by consensus.

3D Morphometry of the Medial versus Lateral Meniscus
3D Morphometric Analysis

The size of the tibial plateau area (ACdAB) of MT and LT was computed after $3 \mathrm{D}$ reconstruction and triangulation (fig. 3), as described previously [Hohe et al., 2002; Wirth et al., 2010a]. Similarly, the 3D size of TA, FA and EA were determined [Wirth et al., 2010a]. The coverage of the tibial plateau area (ACdAB) by TA was determined in absolute $\left(\mathrm{mm}^{2}\right)$ and relative measures (\%), by labeling the $\mathrm{ACdAB}$ surface triangles as covered or uncovered by the MM and LM (fig. 3). A precise technical description of the measurements is given by Wirth et al. [2010a].

As measures of meniscal size, we determined the volume, the mean thickness, the maximal thickness (also termed meniscal height by other authors [Hunter et al., 2006]), and the mean meniscal width, as described previously [Wirth et al., 2010a]. Mean bulging of the meniscus was defined as the average distance 
Fig. 3. $3 \mathrm{D}$ reconstruction of the $\mathrm{MM}$ (green) and LM (red) shown on top of the tibial plateau (blue) from above in a posterior view. Measures of meniscus width and thickness are indicated schematically.
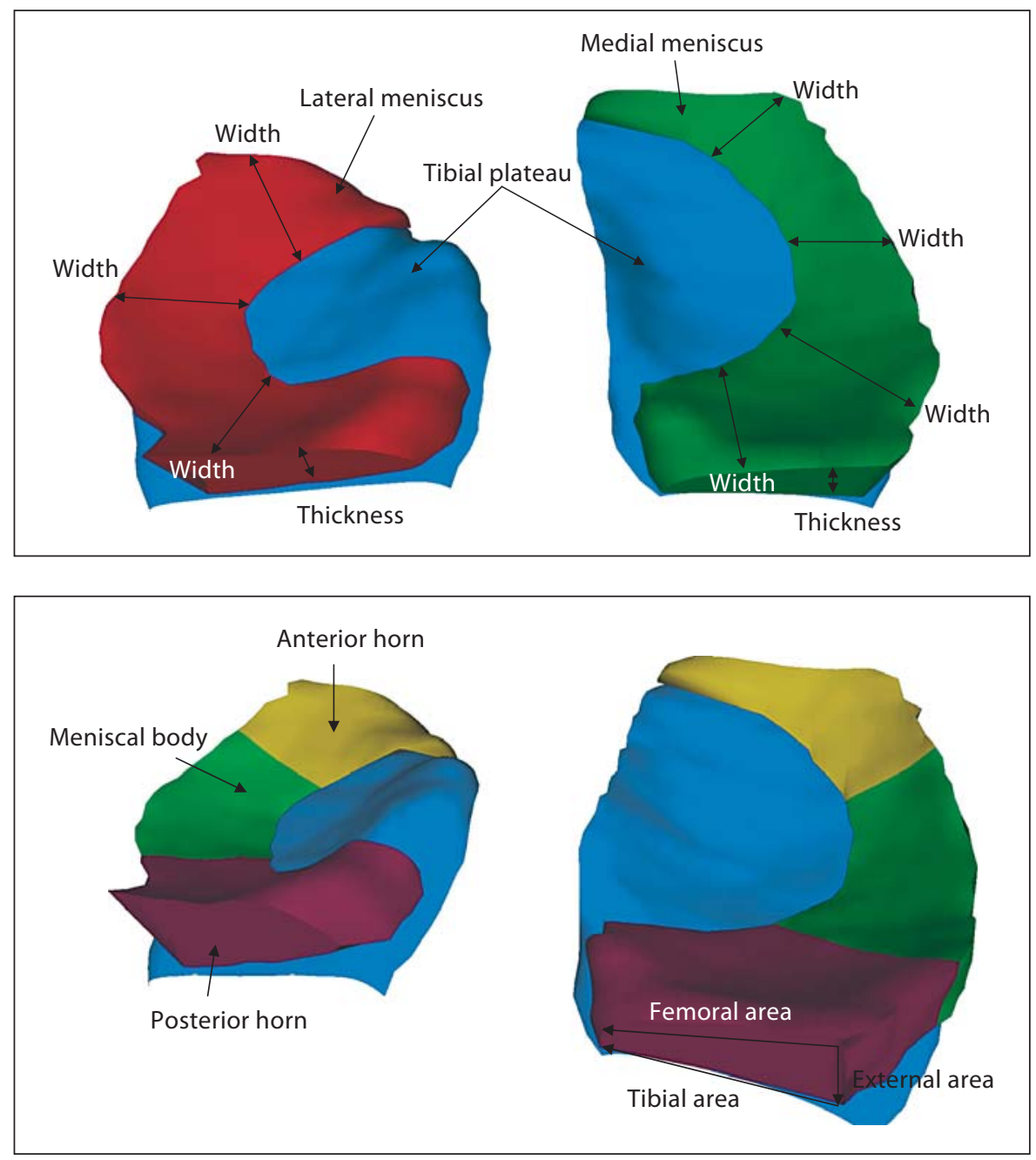

Fig. 4. 3D reconstruction of the $\mathrm{MM}$ (right) and LM (left) subdivided into the anterior (yellow) and posterior (magenta) horns and the meniscal body (green); view from above, showing the tibial coverage of the three meniscal subdivisions. Measures of meniscal areas are indicated schematically. between the EA and a straight line connecting the intersection of TA to EA, and FA to EA, respectively [Wirth et al., 2010a].

As measures of meniscal position (relative to the tibial plateau area), we determined the absolute and relative area of the TA of MM and LM, not covered by the tibial plateau area (ACdAB). Further, we computed the mean and maximal distance of meniscal extrusion as the average and maximal distance between the external margin of the tibial plateau $(\mathrm{ACdAB})$ and the intersection of TA and EA [Wirth et al., 2010a]. Please note that a positive value indicates an 'external' position relative to the external border of the tibial plateau whereas a negative value indicates an 'internal' position relative to the external border of the tibial plateau. The position of the internal margin of the meniscus was determined as the (mean and maximal MM/LM) overlap distance, i.e. the average and maximal distance between the external margin of the tibial plateau $(\mathrm{ACd} A \mathrm{~B})$ and the intersection of TA and FA. Please note that a more negative value indicates a more 'internal' position relative to the external border of the tibial plateau than a less negative value.

In a next step, morphometric measures were computed separately for the body of the meniscus, and the anterior and posterior horns by automated division of the meniscus into these sub- regions [Wirth et al., 2010a] (fig. 4). Analyses in these subregions were limited to measures of thickness and extrusion (see above).

\section{Statistical Analysis and Measurement Reliability}

Mean values and standard deviations (SD) of all the measures of the 47 participants were determined using Microsoft Excel (Microsoft Corporation, Redmond, Wash., USA). Differences between the medial and the lateral femorotibial compartment were assessed using a two-tailed, paired t test, and $\mathrm{p}<0.05$ was considered statistically significant. Since this is an exploratory study, and because the various morphometric measures were interpreted collectively rather than in isolation, no adjustment for parallel testing of multiple morphometric parameters was made.

To evaluate the intra-operator reliability of the segmentations of the OAI reference cohort data, 4 knees were re-analyzed 2 months after the initial segmentation by the same operator (K.B.), without reference to the initial segmentations. The reproducibility of the various morphometric parameters was then expressed as the root mean square SD of the test-retest measurements as suggested by Glüer et al. [1995], and results for the intra-operator reliability are reported in table 1. 
Table 1. Morphometric results for the entire MM and LM

\begin{tabular}{|c|c|c|c|c|c|c|c|c|}
\hline & $\begin{array}{l}\text { Mean } \\
\text { MM }\end{array}$ & $\begin{array}{l}\text { SD } \\
\text { MM }\end{array}$ & $\begin{array}{l}\text { Repro. } \\
\text { MM }\end{array}$ & $\begin{array}{l}\text { Mean } \\
\text { LM }\end{array}$ & $\begin{array}{l}\text { SD } \\
\text { LM }\end{array}$ & $\begin{array}{l}\text { Repro. } \\
\text { LM }\end{array}$ & $\begin{array}{l}\text { Diff. } \\
\%\end{array}$ & $\begin{array}{l}\mathrm{t} \text { test } \\
\text { (paired) }\end{array}$ \\
\hline \multicolumn{9}{|l|}{ Tibial plateau coverage } \\
\hline $\mathrm{ACdAB}, \mathrm{mm}^{2}$ & 1,176 & 122 & 29.5 & 1,100 & 125 & 25.4 & 6.49 & $<0.001$ \\
\hline $\mathrm{ACdAB}_{\mathrm{Cov}}, \mathrm{mm}^{2}$ & 586 & 89.1 & 25.8 & 641 & 79.3 & 16.5 & -9.42 & $<0.001$ \\
\hline $\mathrm{ACdAB}_{\mathrm{Cov}}, \%$ & 49.8 & 5.51 & 1.32 & 58.6 & 6.75 & 0.78 & -17.6 & $<0.001$ \\
\hline $\mathrm{ACdAB}_{\text {Uncov }}, \mathrm{mm}^{2}$ & 590 & 89.0 & 5.80 & 459 & 102 & 12.9 & 22.3 & $<0.001$ \\
\hline $\mathrm{ACdAB}_{\text {Uncov }}, \%$ & 50.2 & 5.51 & 1.32 & 41.4 & 6.75 & 0.78 & 17.4 & $<0.001$ \\
\hline \multicolumn{9}{|l|}{ Meniscus size and shape } \\
\hline Volume, $\mathrm{mm}^{3}$ & 2,438 & 503 & 115 & 2,444 & 472 & 67.7 & -0.26 & 0.924 \\
\hline $\mathrm{TA}, \mathrm{mm}^{2}$ & 625 & 88.3 & 18.5 & 654 & 86.6 & 8.40 & -4.63 & 0.021 \\
\hline $\mathrm{FA}, \mathrm{mm}^{2}$ & 718 & 111 & 13.3 & 750 & 117 & 15.4 & -4.43 & 0.031 \\
\hline $\mathrm{EA}, \mathrm{mm}^{2}$ & 444 & 74.7 & 19.6 & 450 & 67.9 & 6.25 & -1.41 & 0.557 \\
\hline $\mathrm{Th}_{\text {Mean }}, \mathrm{mm}$ & 2.82 & 0.29 & 0.117 & 2.67 & 0.26 & 0.051 & 5.49 & $<0.001$ \\
\hline $\mathrm{Th}_{\mathrm{Max}}, \mathrm{mm}$ & 7.71 & 1.13 & 0.557 & 7.20 & 0.97 & 0.221 & 6.56 & 0.004 \\
\hline Width $_{\text {Mean }}, \mathrm{mm}$ & 9.92 & 1.01 & 0.138 & 10.1 & 1.22 & 0.101 & -1.75 & 0.398 \\
\hline Width $_{\mathrm{Max}}, \mathrm{mm}$ & 18.7 & 1.99 & 0.514 & 14.2 & 2.04 & 0.300 & 24.3 & $<0.001$ \\
\hline $\mathrm{Bul}_{\text {Mean }}, \mathrm{mm}$ & 0.105 & 0.045 & 0.022 & 0.177 & 0.063 & 0.036 & -68.6 & $<0.001$ \\
\hline \multicolumn{9}{|l|}{ Meniscus position } \\
\hline $\mathrm{TA}_{\text {Uncov }}, \mathrm{mm}^{2}$ & 61.2 & 27.4 & 9.39 & 40.0 & 27.1 & 3.45 & 34.7 & $<0.001$ \\
\hline $\mathrm{TA}_{\text {Uncov }}, \%$ & 9.89 & 4.43 & 2.17 & 5.94 & 3.62 & 0.740 & 39.9 & $<0.001$ \\
\hline $\mathrm{mEx}_{\text {Mean }}, \mathrm{mm}$ & 1.19 & 1.26 & 0.502 & -2.56 & 1.24 & 0.581 & 315 & $<0.001$ \\
\hline $\mathrm{mEx}_{\mathrm{Max}}, \mathrm{mm}$ & 7.98 & 1.64 & 0.389 & 8.25 & 1.87 & 0.406 & -3.39 & 0.365 \\
\hline $\mathrm{OvD}_{\text {Mean }}, \mathrm{mm}$ & -12.1 & 1.27 & 0.494 & -17.0 & 2.01 & 0.651 & -39.7 & $<0.001$ \\
\hline $\mathrm{OvD}_{\mathrm{Max}}, \mathrm{mm}$ & -4.70 & 1.43 & 0.415 & -10.3 & 2.02 & 0.705 & -118 & $<0.001$ \\
\hline
\end{tabular}

Repro. = Intra-operator reproducibility, as determined by the root mean square SD for test-retest in 4 knees; Diff. = difference; $\mathrm{ACdAB}_{\mathrm{Cov}} / \mathrm{ACdAB}_{\text {Uncov }}=\mathrm{ACdAB}$ covered/uncovered with meniscus; Th = thickness of the menisci; Width = width of the menisci; $\mathrm{TA}_{\text {Uncov }}=\mathrm{TA}$ of the meniscus not covering the tibial platea; $\mathrm{Ex}=$ extrusion; OvD = overlap distance; Bul $\mathrm{Mean}=$ mean bulging of the meniscus; Max $_{\text {maximal. }}$.

\section{Results}

\section{Entire MM versus $L M$}

The cartilage surface $(\mathrm{ACdAB})$ of the MT plateau $(11.8$ $\left.\pm 1.2 \mathrm{~cm}^{2}\right)$ was significantly larger $(+6.5 \%$; $<<0.001)$ than that for the LT $\left(11.0 \pm 1.3 \mathrm{~cm}^{2}\right.$; table 1$)$. Whereas $58.6 \pm$ $6.8 \%$ of the LT was covered with the LM, only $49.8 \pm 5.5 \%$ of the MT was covered with the MM ( $<<0.001)$. Accordingly, the surface of the tibial plateau uncovered with meniscus was greater for the MM than for the LM (table 1). In the 47 cases examined, medial coverage was greater than lateral coverage in only 3 cases and medial and lateral coverage was similar in 3 cases. In no case, a discoid meniscus was observed either medially or laterally.

The volume of the $\operatorname{LM}(2.444 \pm 0.47 \mathrm{ml})$ was very similar to that of the MM $(2.438 \pm 0.50 \mathrm{ml} ; \mathrm{p}=0.924$; table 1). However, the LM displayed significantly greater TA and FA than the MM (table 1), whereas the mean MM thickness $(2.82 \pm 0.3 \mathrm{~mm})$ was significantly greater $(\mathrm{p}<$
$0.001)$ than that of the $\operatorname{LM}(2.67 \pm 0.3 \mathrm{~mm})$. The same applied for the maximal thickness $(7.7 \pm 1.3 \mathrm{~mm}$ for MM vs. $7.2 \pm 1.0 \mathrm{~mm}$ for LM; p $<0.01$; table 1$)$. The mean LM width $(10.1 \pm 1.2 \mathrm{~mm})$ was not significantly $(\mathrm{p}=0.398)$ different from that of the MM $(9.9 \pm 1.0 \mathrm{~mm})$, but the maximal width was $24 \%$ greater in the MM than in the LM ( $\mathrm{p}<0.001$; table 1). In both MM and LM, FA was greater than TA, and both were greater than EA (table 1). EA displayed a larger degree of bulging $(0.18 \pm 0.06 \mathrm{~mm})$ in the LM than in the MM $(0.11 \pm 0.05 \mathrm{~mm}$; $<<0.001)$.

In the LM, $5.9 \pm 3.6 \%$ of TA was uncovered by the tibial plateau area, whereas in the MM a significantly $(\mathrm{p}<0.001)$ larger percentage of the TA $(9.9 \pm 4.4 \%)$ was uncovered by MT (table 1) due to stronger extrusion. Amongst the 47 cases examined, the uncovered area of the LM was greater than that of the MM in only 12 cases. Accordingly, the mean extrusion distance, i.e. the distance between the external margin of the tibial plateau and the intersection of the TA and EA, was also less for 
Table 2. Morphometric results for the body, anterior horn and posterior horn of the MM and LM

\begin{tabular}{|c|c|c|c|c|c|c|c|c|}
\hline & $\begin{array}{l}\text { Mean } \\
\text { MM }\end{array}$ & $\begin{array}{l}\text { SD } \\
\mathrm{MM}\end{array}$ & $\begin{array}{l}\text { Repro. } \\
\text { MM }\end{array}$ & $\begin{array}{l}\text { Mean } \\
\text { LM }\end{array}$ & $\begin{array}{l}\text { SD } \\
\text { LM }\end{array}$ & $\begin{array}{l}\text { Repro. } \\
\text { LM }\end{array}$ & $\begin{array}{l}\text { Diff. } \\
\%\end{array}$ & $\begin{array}{l}\mathrm{t} \text { test } \\
\text { (paired) }\end{array}$ \\
\hline \multicolumn{9}{|l|}{ Meniscal body } \\
\hline $\mathrm{Th}_{\text {Mean }}, \mathrm{mm}$ & 2.56 & 0.257 & 0.092 & 2.59 & 0.353 & 0.079 & -1.1 & 0.569 \\
\hline $\mathrm{Th}_{\mathrm{Max}}, \mathrm{mm}$ & 6.37 & 0.717 & 0.572 & 6.72 & 0.910 & 0.201 & -5.4 & 0.017 \\
\hline $\mathrm{Bul}_{\text {Mean }}, \mathrm{mm}$ & 0.085 & 0.048 & 0.018 & 0.170 & 0.099 & 0.039 & -102 & $<0.001$ \\
\hline $\mathrm{mEx}_{\text {Mean }}, \mathrm{mm}$ & 1.20 & 1.53 & 0.548 & -2.87 & 1.36 & 0.464 & - & $<0.001$ \\
\hline $\operatorname{mEx}_{\mathrm{Max}}, \mathrm{mm}$ & 5.71 & 1.13 & 0.529 & 2.67 & 3.21 & 1.11 & 53.3 & $<0.001$ \\
\hline \multicolumn{9}{|c|}{ Meniscal anterior horn } \\
\hline $\mathrm{Th}_{\text {Mean }}, \mathrm{mm}$ & 2.41 & 0.292 & 0.133 & 2.42 & 0.326 & 0.049 & -0.3 & 0.910 \\
\hline $\mathrm{Th}_{\mathrm{Max}}, \mathrm{mm}$ & 6.16 & 0.826 & 0.360 & 6.56 & 0.958 & 0.162 & -6.5 & 0.007 \\
\hline $\mathrm{Bul}_{\text {Mean }}, \mathrm{mm}$ & 0.10 & 0.070 & 0.029 & 0.149 & 0.079 & 0.046 & -44.2 & 0.001 \\
\hline $\mathrm{mEx}_{\text {Mean }}, \mathrm{mm}$ & 2.92 & 1.18 & 0.269 & -2.17 & 1.99 & 1.19 & - & $<0.001$ \\
\hline $\mathrm{mEx}_{\mathrm{Max}}, \mathrm{mm}$ & 6.34 & 0.903 & 0.152 & 3.53 & 3.24 & 0.794 & 44.3 & $<0.001$ \\
\hline \multicolumn{9}{|c|}{ Meniscal posterior horn } \\
\hline $\mathrm{Th}_{\text {Mean }}, \mathrm{mm}$ & 3.11 & 0.375 & 0.149 & 2.88 & 0.352 & 0.056 & 7.6 & $<0.001$ \\
\hline $\mathrm{Th}_{\mathrm{Max}}, \mathrm{mm}$ & 7.63 & 1.21 & 0.557 & 6.74 & 1.03 & 0.205 & 11.6 & $<0.001$ \\
\hline $\mathrm{Bul}_{\text {Mean }}, \mathrm{mm}$ & 0.144 & 0.068 & 0.033 & 0.209 & 0.138 & 0.072 & -45.3 & 0.005 \\
\hline $\mathrm{mEx}_{\text {Mean }}, \mathrm{mm}$ & -0.520 & 2.32 & 0.698 & -2.44 & 1.52 & 0.661 & - & $<0.001$ \\
\hline $\operatorname{mEx}_{\text {Max }}, \mathrm{mm}$ & 7.11 & 2.97 & 0.389 & 7.62 & 3.14 & 0.406 & -7.2 & 0.376 \\
\hline
\end{tabular}

Repro. = Intra-operator reproducibility, as determined by the root mean square SD for test-retest in 4 knees; Diff. = difference;

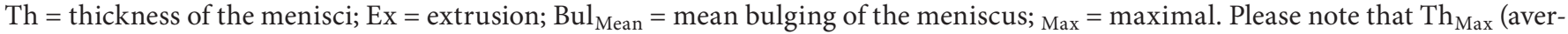
aged across all subjects) is smaller in the subregions than in the entire meniscus, because $\mathrm{Th}_{\mathrm{Max}}$ was not located in the same subregion in each participant.

$\operatorname{LM}(-2.56 \pm 1.24 \mathrm{~mm})$ than for MM $(1.19 \pm 1.26 \mathrm{~mm}$; table 1$)$ and even pointed in the opposite direction (i.e. 'intrusion'). The maximal extrusion, in contrast, did not differ significantly between MM and LM (table 1 ). The mean overlap distance was more negative $(p<0.001)$, i.e. located more internally for the LM $(-17.0 \pm 2.0 \mathrm{~mm})$ than for the MM (-12.1 $\pm 1.3 \mathrm{~mm})$, and the same applied for the maximal overlap distance (table 1).

\section{Meniscus Subregions (Anterior and Posterior Horn, and Body)}

In the meniscal body, the mean LM thickness (2.59 \pm $0.4 \mathrm{~mm}$ ) did not differ significantly from that of $\mathrm{MM}$ (2.56 $\pm 0.3 \mathrm{~mm} ; \mathrm{p}=0.569)$, whereas the maximal $\mathrm{LM}$ thickness $(6.72 \pm 0.9 \mathrm{~mm})$ exceeded that of MM (6.37 \pm $0.7 \mathrm{~mm} ; \mathrm{p}=0.017$; table 2). Note that this is opposite to the maximal thickness of the entire meniscus, which was significantly greater medially than laterally (see above). In the anterior horn, the maximal thickness was also greater laterally than medially, whereas in the posterior horn the opposite was observed, both for the mean and maximal thickness (table 2). Generally, the (mean and maximal) thickness in the posterior horn exceeded that in the body, and that in the body exceeded that in the an- terior horn. Note that the (average) maximal thickness of the entire meniscus exceeded those in the meniscus subregions (across all participants), because the maximum was not located in the same subregion in all participants. Bulging was largest in the posterior horn (both in MM and LM) and was significantly greater in LM than in MM in all 3 subregions (table 2).

Physiological extrusion over the entire meniscus subregion (mean extrusion) was only observed in the anterior horn and body of the MM (but not in the LM), whereas some extrusion (parts of the subregion, i.e. the maximum) was observed in all subregions of the MM and LM (maximal distance of meniscal extrusion). Extrusion values differed significantly between all MM and LM subregions (table 2) and amounted to $1.20 \pm 1.53 \mathrm{~mm}$ for the $\mathrm{MM}$ and to $-2.87 \pm 1.36 \mathrm{~mm}$ for the $\mathrm{LM}$.

\section{Discussion}

To our knowledge, this is the first study to directly compare morphological measures of the human MM and LM in healthy adult knees without OA, based on in vivo imaging data. Specifically, we explored the size, thickness 
and relative position of the MM and LM to the MT and LT cartilage. Our results show that the LM covers a significantly greater area of the ipsilateral tibia than the MM. While the volume of the LM and MM were very similar, the LM had somewhat larger surface areas (albeit a smaller maximal width) than the MM, and a smaller mean and maximal thickness than the MM. This applied in particular to the posterior horn, which generally displayed a greater thickness than the body and anterior horn in both menisci. Whereas the MM displayed some (physiological) extrusion, its external margin extending beyond the external margin of the MT, the LM rather displayed a physiological 'intrusion', i.e. its external margin was on average located internally to the external margin of the LT. The greatest physiological extrusion of the MM was observed in the anterior horn, and the greatest 'intrusion' of the LM in the body.

The OAI data were used because the inclusion criteria for the reference cohort were very rigorous, not only excluding subjects with frequent or infrequent knee pain and radiographic signs of knee OA, but also subjects with potential risk factors for degenerative joint disease. DESSwe was used for segmentation, because of its high resolution $(0.7-\mathrm{mm}$ slices, reformatted to $1.5-\mathrm{mm}$ reconstructed double oblique coronal images, with an $0.37-\mathrm{mm}$ in-plane resolution), whereas the coronal IW TSE, which is commonly used to evaluate meniscal pathology [Roemer et al., 2005; Guermazi et al., 2008; Roemer et al., 2009], was acquired by the OAI using a 3-mm slice thickness. Since geometric measurements were performed, the voxel size is critical in obtaining accurate and precise information. Opposite to the coronal fast-low angle shot acquisition of the OAI that also provides high-resolution images but is $T_{1}$ weighted, the DESS has $\mathrm{T}_{2^{-}}$and $\mathrm{T}_{2}{ }^{*}$ weighted signals that contribute to the combined DESS signal [Eckstein et al., 2006b], enabling a clear delineation of the meniscal tissue. Another $\mathrm{T}_{2}{ }^{*}$-weighted gradient echo sequence was previously used by Bowers et al. [2007] and the authors reported satisfactory test-retest precision and good agreement between MRI-based meniscal volume measurements in cadaver specimens and water displacement of surgically removed menisci. However, because the IW TSE sequence represents a gold standard in the clinical evaluation of the meniscus [Roemer et al., 2005; Guermazi et al., 2008; Roemer et al., 2009], we additionally used these images to assist in the readings of the DESS, by displaying them in parallel with the latter during the segmentation process, particularly in situations of unclear contrast. Moreover, DESSwe has the advantage that it more clearly delineated the cartilage sur- face of the tibial plateaus than the IW TSE and that it has been cross-calibrated previously [Eckstein et al., 2006b; Wirth et al., 2010b] with validated MRI sequences for measurement of cartilage thickness and surface areas [Burgkart et al., 2001; Graichen et al., 2004]. Precise delineation of the cartilage surface is particularly crucial in the context of meniscus extrusion evaluation.

Limitations of the current study are the limited sample size. However, a paired t test was applied to evaluate differences between the MM and LM in the same subjects, which is more powerful (and requires smaller samples) than unpaired comparisons between different subjects. Another limitation is the use of coronal slices; these are ideal for evaluating the meniscal body, but display partial volume effects in anterior and posterior horns and thus preclude accurate measurements of anterior and posterior extrusion, as previously described (using sagittal slices) by Hunter et al. [2006]. For the same reason, the volumes reported may be smaller than the true meniscal values, as tissue that was potentially overlapping the anterior and posterior margins of the tibial plateau area was not included in the analysis due to the reasons mentioned above. Finally, MRI were acquired in a supine position without weight bearing, whereas the meniscus has its primary biomechanical function during load bearing. However, MRI were acquired in a highly standardized position, i.e. $10^{\circ}$ flexion [Eckstein et al., 2007a], and a recent study showed that there were only minimal differences in the morphology and relative position of the meniscus between non-weight-bearing and weight-bearing MRI when performing analysis of MRI obtained using an MRI-compatible loading device [Frobell et al., 2009]. Using ultrasound, Verdonk et al., [2004] also did not find significant differences of LM extrusion between a supine (non-weight-bearing) position, bipodal stance and unipodal stance in 10 adults.

As previously shown using spin echo images [Wirth et al., 2010a], the image analysis technique used here for the quantitative 3D analysis of meniscus size, shape and position also shows good intra-operator test-retest reliability in the current study, using the DESSwe images. For most parameters, the test-retest (root mean square) SD was considerably lower than the intersubject variability across the participants examined. Further, the image analysis technique used permits fully automated separation of the meniscus into the body, the anterior horn and the posterior horn [Wirth et al., 2010a]. Swanson et al. [2010] recently presented a technique for semi-automated segmentation of the meniscus in the OAI reference cohort, but the authors only evaluated the LM (but not the MM) 
and did not provide quantitative morphometric results. Elsner et al. [2010] also performed morphometric measurements of the meniscus in the OAI reference cohort, but only studied the MM (but not the LM).

The morphometric values reported in the current paper are in reasonable agreement with those of the small non-arthritic reference cohort studied by Wirth et al. [2010a], with values for MM size being somewhat greater, one possible explanation being that Wirth et al. [2010a] studied women, whereas we studied men. Hunter et al. [2006] reported a height of $5.4 \pm 2.5 \mathrm{~mm}$ for the LM in a central coronal image, which corresponds well with our measurements of maximal meniscus thickness in the body of the LM. In contrast, the authors [Hunter et al., 2006] reported a height of only $2.9 \pm 2.0 \mathrm{~mm}$ for the $\mathrm{MM}$, whereas our values are more than twice of that. The participants studied by Hunter et al. [2006], however, had radiographic evidence of $\mathrm{OA}$ of the knee, with a greater proportion of medial than lateral femorotibial OA, and on average with various degrees of malalignment. Likely, therefore, the morphology of the meniscus was structurally abnormal, particularly on the medial side. Our results are also in some contrast with assumptions made by Chivers and Howitt [2009], who reported the MM to be thinner than the LM. Whereas we also found a greater (maximal) thickness of the MM in the body and anterior horn, the mean and maximal thickness of the posterior horn and the entire meniscus were greater laterally than medially.

Partly contradictory assumptions have been published with regard to how much of the tibial plateau is physiologically covered by the MM and LM, respectively (i.e. $50 \%$ of the MT and $70 \%$ of the LT surface by Rath and Richmond [2000], or $60 \%$ of the medial and $80 \%$ of the lateral plateau by Clark and Ogden [1983]. To our knowledge, the only study that performed actual quantitative measurements of tibial plateau coverage was that by $\mathrm{Fu}$ kazawa et al. [2009], who reported 59\% meniscal coverage medially and $75 \%$ laterally. A potential explanation why we found smaller values (49\% medially and 59\% laterally) may be that these authors [Fukazawa et al., 2009] used a projectional image for quantitative determining the ratio of covered and uncovered areas, which underestimates the (internal) uncovered part of the tibial plateau that ascends towards the intercondylar area, is oblique to the image plane and is therefore underestimated in a projectional image. Also, the authors performed measurements in cadaver joints in which disarticulation may have disturbed the normal anatomy, whereas our measurement was performed in intact joints in vivo. In contrast to $\mathrm{Fu}$ - kazawa et al. [2009], in our patients, FA (and TA) of the LM were significantly larger than those of the MM, whereas they reported similar values for MM and LM. In agreement with Fukazawa et al. [2009], the MT plateau area was also significantly larger in our cohort than that of the LT (ratio 1.06:1). Eckstein et al. [2009] reported the ratio of the MT versus LT plateau area to depend on the alignment of the lower limb, with a 1.18:1 ratio in neutral knees, 1.28:1 in varus knees and 1.13:1 in valgus knees. This study [Eckstein et al., 2009], however, was performed in patients with moderate-severe OA, whereas the current study was performed in knees without (risk factors of) OA.

In conclusion, our results show that the LM covers a significantly greater area of the LT (59\%) than the MM does of the MT (50\%). The volumes of the LM and MM were similar, but the LM displayed about $4.5 \%$ larger surface areas than the MM, and (on average) an approximately $6 \%$ smaller mean and maximal thickness than the MM. The posterior horn displayed a greater thickness than the body and anterior horn in both menisci. Whereas the MM displayed some (physiological) extrusion of $1.2 \mathrm{~mm}$ on average, the external margin of the LM was located internally ('intrusion') to the rim of the LT ( -2.6 $\mathrm{mm}$ on average). The greatest physiological extrusion of the MM was observed in the anterior horn, and the greatest 'intrusion' of the LM in the body. These data may guide biomechanical experimentation and theory that explores form-function relationships of the femorotibial joint as well as strategies for meniscal tissue engineering and transplantation in an attempt to restore normal joint conditions.

\section{Acknowledgments}

We would like to thank Andrea Wenger for the support in establishing the reading rules for meniscal segmentation. We would also like to thank the OAI participants, investigators and technicians for generating this publicly available image data set. The image acquisition was funded by the OAI, a public-private partnership comprised of five contracts (N01-AR-2-2258; N01AR-2-2259; N01-AR-2-2260; N01-AR-2-2261, and N01-AR-22262) funded by the National Institutes of Health, a branch of the Department of Health and Human Services, and conducted by the OAI Study Investigators. Private funding partners include Merck Research Laboratories; Novartis Pharmaceuticals Corporation, GlaxoSmithKline, and Pfizer, Inc. Private sector funding for the OAI is managed by the Foundation for the $\mathrm{Na}$ tional Institutes of Health. The image analysis was performed without funding support. 


\section{References}

Baker, B.M., A.O. Gee, N.P. Sheth, G.R. Huffman, B.J. Sennett, T.P. Schaer, R.L. Mauck (2009) Meniscus tissue engineering on the nanoscale: from basic principles to clinical application. J Knee Surg 22: 45-59.

Bowers, M.E., G.A. Tung, B.C. Fleming, J.J. Crisco, J. Rey (2007) Quantification of meniscal volume by segmentation of $3 \mathrm{~T}$ magnetic resonance images. J Biomech 40: 2811-2815.

Burgkart, R., F. Eckstein, H. Sittek, I. Schittich, J. Träger, E. Hipp (1995a) Schnittanatomie des Kniegelenks - Korrelation von anatomischem Präparat, Computertomograhie und Magnetresonanztomographie, Schwerpunkt: hinteres Kreuzband und mediales Kompartiment. Sport Orthop Traumatol 11.4: 262-267.

Burgkart, R., C. Glaser, A. Hyhlik-Durr, K.H. Englmeier, M. Reiser, F. Eckstein (2001) Magnetic resonance imaging-based assessment of cartilage loss in severe osteoarthritis: accuracy, precision, and diagnostic value. Arthritis Rheum 44: 2072-2077.

Burgkart, R., R. Schelter, F. Eckstein, H. Rechl, J. Träger (1995b) Schnittanatomie des Kniegelenks - Korrelation von anatomischem Präparat, Computertomograhie und Magnetresonanztomographie, Schwerpunkt: vorderes Kreuzband. Sport Orthop Traumatol 11.1: 46-52.

Burgkart, R., R. Schelter, F. Eckstein, H. Rechl, J. Träger (1995c) Schnittanatomie des Kniegelenks - Korrelation von anatomischem Präparat, Computertomographie und Magnetresonanztomographie, Schwerpunkt: posterolaterale Knieregion. Sport Orthop Traumatol 11.2: 112-117.

Burgkart, R., R. Schelter, F. Eckstein, H. Sittek, I. Schittich, J. Träger (1995d) Schnittanatomie des Kniegelenks - Korrelation von anatomischem Präparat, Computertomographie und Magnetresonanztomographie, Schwerpunkt: posterolaterale Knieregion II. Sport Orthop Traumatol 11.3: 188-192.

Chivers, M.D., S.D. Howitt (2009) Anatomy and physical examination of the knee menisci: a narrative review of the orthopedic literature. J Can Chiropr Assoc 53: 319-333.

-Clark, C.R., J.A Ogden (1983) Development of the menisci of the human knee joint. Morphological changes and their potential role in childhood meniscal injury. J Bone Joint Surg Am 65: 538-547.

Conaghan, P.G., D.T. Felson (2004) Structural associations of osteoarthritis pain: lessons from magnetic resonance imaging. Novartis Found Symp 260: 191-201, discussion 201205, 277-279.

Costa, C.R., W.B. Morrison, J.A. Carrino (2004) Medial meniscus extrusion on knee MRI: is extent associated with severity of degeneration or type of tear? AJR Am J Roentgenol 183: $17-23$.

3D Morphometry of the Medial versus Lateral Meniscus
Doskocil, M. (1984) Study of the development of the human knee joint (in German). Anat Anz 157: 35-41.

Eckstein, F., G. Ateshian, R. Burgkart, D. Burstein, F. Cicuttini, B. Dardzinski, M. Gray, T.M. Link, S. Majumdar, T. Mosher, P.C. Peterfy, S. Totterman, J. Waterton, C.S. Winalski, D. Felson (2006a) Proposal for a nomenclature for magnetic resonance imaging based measures of articular cartilage in osteoarthritis. Osteoarthritis Cartilage 14: 974-983.

Eckstein, F., M. Hudelmaier, S. Cahue, M. Marshall, L. Sharma (2009) Medial-to-lateral ratio of tibiofemoral subchondral bone area is adapted to alignment and mechanical load. Calcif Tissue Int 84: 186-194.

Eckstein, F., M. Hudelmaier, W. Wirth, B. Kiefer, R. Jackson, J. Yu, C.B. Eaton, E. Schneider (2006b) Double echo steady state magnetic resonance imaging of knee articular cartilage at 3 Tesla: a pilot study for the Osteoarthritis Initiative. Ann Rheum Dis 65: 433441.

Eckstein, F., M. Kunz, M. Hudelmaier, R. Jackson, J. Yu, C.B. Eaton, E. Schneider (2007a) Impact of coil design on the contrast-tonoise ratio, precision, and consistency of quantitative cartilage morphometry at 3 Tesla: a pilot study for the osteoarthritis initiative. Magn Reson Med 57: 448-454.

Eckstein, F., M. Kunz, M. Schutzer, M. Hudelmaier, R.D. Jackson, J. Yu, C.B. Eaton, E. Schneider (2007b) Two year longitudinal change and test-retest-precision of knee cartilage morphology in a pilot study for the osteoarthritis initiative. Osteoarthritis Cartilage 15: 1326-1332.

Eisenhart-Rothe, R., C. Bringmann, M. Siebert, M. Reiser, K.H. Englmeier, F. Eckstein, H. Graichen (2004) Femoro-tibial and menisco-tibial translation patterns in patients with unilateral anterior cruciate ligament deficiency - a potential cause of secondary meniscal tears. J Orthop Res 22: 275-282.

Elsner, J.J., S. Portnoy, F. Guilak, A. Shterling, E. Linder-Ganz (2010) MRI-based characterization of bone anatomy in the human knee for size matching of a medial meniscal implant. J Biomech Eng 132: 101008.

Englund, M., A. Guermazi, S.L. Lohmander (2009) The role of the meniscus in knee osteoarthritis: a cause or consequence? Radiol Clin North Am 47: 703-712.

Frobell, R.B., R.B. Souza, B.T. Wyman, W. Wirth, M.P. Hellio-Le Graverand, M. Hudelmaier, X. Li, T. Link, F. Eckstein, S. Majumdar (2009) Meniscus shape, position, and signal under simulated weightbearing and nonweightbearing conditions in vivo. Osteoarthritis Cartilage 17(suppl 1): abstract S236.

-Fukazawa, I., T. Hatta, Y. Uchio, H. Otani (2009) Development of the meniscus of the knee joint in human fetuses. Congenit Anom (Kyoto) 49: 27-32.
Glowacki, J. (2001) Engineered cartilage, bone, joints, and menisci. Potential for temporomandibular joint reconstruction. Cells Tissues Organs 169: 302-308.

Glüer, C.C., G. Blake, Y. Lu, B.A. Blunt, M. Jergas, H.K. Genant (1995) Accurate assessment of precision errors: how to measure the reproducibility of bone densitometry techniques. Osteoporos Int 5: 262-270.

Graichen, H., R. Eisenhart-Rothe, T. Vogl, K.H. Englmeier, F. Eckstein (2004) Quantitative assessment of cartilage status in osteoarthritis by quantitative magnetic resonance imaging: technical validation for use in analysis of cartilage volume and further morphologic parameters. Arthritis Rheum 50: 811-816.

Guermazi, A., D. Burstein, P. Conaghan, F. Eckstein, M.P. Hellio Le Graverand-Gastineau, H. Keen, F.W. Roemer (2008) Imaging in osteoarthritis. Rheum Dis Clin North Am 34: 645-687.

Hardy, P.A., M.P. Recht, D. Piraino, D. Thomasson (1996) Optimization of a dual echo in the steady state (DESS) free-precession sequence for imaging cartilage. J Magn Reson Imaging 6: 329-335.

Haut, T.L., M.L. Hull, S.M. Howell (1998) A high-accuracy three-dimensional coordinate digitizing system for reconstructing the geometry of diarthrodial joints. J Biomech 31: 571-577.

Hohe, J., G. Ateshian, M. Reiser, K.H. Englmeier, F. Eckstein (2002) Surface size, curvature analysis, and assessment of knee joint incongruity with MRI in vivo. Magn Reson Med 47: 554-561.

-Hunter, D.J., Y.Q. Zhang, J.B. Niu, X. Tu, S. Amin, M. Clancy, A. Guermazi, M. Grigorian, D. Gale, D.T. Felson (2006) The association of meniscal pathologic changes with cartilage loss in symptomatic knee osteoarthritis. Arthritis Rheum 54: 795-801.

Kornaat, P.R., J.L. Bloem, R.Y. Ceulemans, N. Riyazi, F.R. Rosendaal, R.G. Nelissen, W.O. Carter, M.P. Hellio Le Graverand, M. Kloppenburg (2006) Osteoarthritis of the knee: association between clinical features and MR imaging findings. Radiology 239: 811817.

Kramer, D.E., L.J. Micheli (2009) Meniscal tears and discoid meniscus in children: diagnosis and treatment. J Am Acad Orthop Surg 17: 698-707.

Kurosawa, H., T. Fukubayashi, H. Nakajima H (1980) Load-bearing mode of the knee joint: physical behavior of the knee joint with or without menisci. Clin Orthop Relat Res 283290.

Lee, B.I., Y.S. Lee, S.W. Kwon, S.W. Choi, K.H. Cho, Y.J. Kwon (2007) Bilateral symptomatic discoid medial meniscus: report of three cases. Knee Surg Sports Traumatol Arthrosc 15: 739-743. 
-Lerer, D.B., H.R. Umans, M.X. Hu, M.H. Jones (2004) The role of meniscal root pathology and radial meniscal tear in medial meniscal extrusion. Skeletal Radiol 33: 569-574.

-Magee, T (2008) MR findings of meniscal extrusion correlated with arthroscopy. J Magn Reson Imaging 28: 466-470.

McDermott, I.D., F. Sharifi, A.M. Bull, C.M. Gupte, R.W. Thomas, A.A. Amis (2004) An anatomical study of meniscal allograft sizing. Knee Surg Sports Traumatol Arthrosc 12: $130-135$.

Meller, R., F. Schiborra, G. Brandes, K. Knobloch, T. Tschernig, S. Hankemeier, C. Haasper, A. Schmiedl, M. Jagodzinski, C. Krettek, E. Willbold (2009) Postnatal maturation of tendon, cruciate ligament, meniscus and articular cartilage: a histological study in sheep. Ann Anat 191: 575-585.

-Messner, K., J. Gao (1998) The menisci of the knee joint. Anatomical and functional characteristics, and a rationale for clinical treatment. J Anat 193: 161-178.

Murlimanju, B.V., N. Nair, M.M. Pai, A. Krishnamurthy, P.X. Chandra (2010) Morphology of the medial meniscus of the knee in human fetuses. Rom J Morphol Embryol 51: 347351.

Peterfy, C., J. Li, S. Zaim, J. Duryea, J. Lynch, Y. Miaux, W. Yu, H.K. Genant (2003) Comparison of fixed-flexion positioning with fluoroscopic semi-flexed positioning for quantifying radiographic joint-space width in the knee: test-retest reproducibility. Skeletal Radiol 32: 128-132.

Peterfy, C.G., E. Schneider, M. Nevitt (2008) The osteoarthritis initiative: report on the design rationale for the magnetic resonance imaging protocol for the knee. Osteoarthritis Cartilage 16: 1433-1441.

- Petersen, W., B. Tillmann (1998) Collagenous fibril texture of the human knee joint menisci. Anat Embryol (Berl) 197: 317-324.

Pollard, M.E., Q. Kang, E.E. Berg (1995) Radiographic sizing for meniscal transplantation. Arthroscopy 11: 684-687.
Rath, E., J.C. Richmond (2000) The menisci: basic science and advances in treatment. $\mathrm{Br} \mathrm{J}$ Sports Med 34: 252-257.

Rennie, W.J., D.B. Finlay (2006) Meniscal extrusion in young athletes: associated knee joint abnormalities. AJR Am J Roentgenol 186: 791-794.

Roemer, F.W., A. Guermazi, J.A. Lynch, C.G. Peterfy, M.C. Nevitt, N. Webb, J. Li, A. Mohr, H.K. Genant, D.T. Felson (2005) Short tau inversion recovery and proton density-weighted fat suppressed sequences for the evaluation of osteoarthritis of the knee with a $1.0 \mathrm{~T}$ dedicated extremity MRI: development of a time-efficient sequence protocol. Eur Radiol 15: 978-987.

Roemer, F.W., Y. Zhang, J. Niu, J.A. Lynch, M.D. Crema, M.D. Marra, M.C. Nevitt, D.T. Felson, L.B. Hughes, G.Y. El-Khoury, M. Englund, A. Guermazi (2009) Tibiofemoral joint osteoarthritis: risk factors for MR-depicted fast cartilage loss over a 30-month period in the multicenter osteoarthritis study. Radiology 252: 772-780.

Schneider, E., M. NessAiver, D. White, D. Purdy, L. Martin, L. Fanella, D. Davis, M. Vignone, G. Wu, R. Gullapalli (2008) The osteoarthritis initiative (OAI) magnetic resonance imaging quality assurance methods and results. Osteoarthritis Cartilage 16: 994-1004.

Stone, K.R., A. Freyer, T. Turek, A.W. Walgenbach, S. Wadhwa, J. Crues (2007) Meniscal sizing based on gender, height, and weight. Arthroscopy 23: 503-508.

-Stone, K.R., D.W. Stoller, S.G. Irving, C. Elmquist, G. Gildengorin (1994) 3D MRI volume sizing of knee meniscus cartilage. Arthroscopy 10: 641-644.

Swanson, M.S., J.W. Prescott, T.M. Best, K. Powell, R.D. Jackson, F. Haq, M.N. Gurcan (2010) Semi-automated segmentation to assess the lateral meniscus in normal and osteoarthritic knees. Osteoarthritis Cartilage 18: 344353.

Tachibana, Y., Y. Yamazaki, S. Ninomiya (2003) Discoid medial meniscus. Arthroscopy 19: E12-E18.
Torres, L., D.D. Dunlop, C. Peterfy, A. Guermazi, P. Prasad, K.W. Hayes, J. Song, S. Cahue, A. Chang, M. Marshall, L. Sharma (2006) The relationship between specific tissue lesions and pain severity in persons with knee osteoarthritis. Osteoarthritis Cartilage 14: 10331040.

Van Thiel, G.S., N. Verma, A. Yanke, S. Basu, J. Farr, B. Cole (2009) Meniscal allograft size can be predicted by height, weight, and gender. Arthroscopy 25: 722-727.

Verdonk, P., Y. Depaepe, S. Desmyter, M. De Muynck, K.F. Almqvist, K. Verstraete, R. Verdonk (2004) Normal and transplanted lateral knee menisci: evaluation of extrusion using magnetic resonance imaging and ultrasound. Knee Surg Sports Traumatol Arthrosc 12: 411-419.

Vidyadhara, S., S.K. Rao, S. Rao (2006) Discoid medial meniscus. Varied presentation of 3 knees. Saudi Med J 27: 888-891.

Walker, P.S., M.J. Erkman (1975) The role of the menisci in force transmission across the knee. Clin Orthop Relat Res 184-192.

Wirth, W., R.B. Frobell, R.B. Souza, X. Li, B.T. Wyman, M.P. Le Graverand, T.M. Link, S. Majumdar, F. Eckstein (2010a) A three-dimensional quantitative method to measure meniscus shape, position, and signal intensity using MR images: a pilot study and preliminary results in knee osteoarthritis. Magn Reson Med 63: 1162-1171.

Wirth, W., M. Nevitt, M.P. Hellio Le Graverand, O. Benichou, D. Dreher, R.Y. Davies, J. Lee, K. Picha, A. Gimona, S. Maschek, M. Hudelmaier, F. Eckstein (2010b) Sensitivity to change of cartilage morphometry using coronal FLASH, sagittal DESS, and coronal MPR DESS protocols - comparative data from the Osteoarthritis Initiative (OAI). Osteoarthritis Cartilage 18: 547-554.

Yang, P.J., J.S. Temenoff (2009) Engineering orthopedic tissue interfaces. Tissue Eng Part B Rev 15: 127-141. 